\title{
Evaluation Concept of Measurement Results in Utility Meters Testing
}

\author{
Peter Trebuňa ${ }^{1, *}$, Jaromír Markovič ${ }^{2}$, Štefan Král ${ }^{2}$, Tomáš Švantner ${ }^{2}$, Ladislav Rosocha ${ }^{1}$ \\ Technical University of Košice, Faculty of Mechanical Engineering, Institute of Management, Industrial and Digital Engineering, Slovakia \\ Slovak Legal Metrology, Hviezdoslavova 31, Banská Bystrica, Slovakia
}

\begin{abstract}
The conformity assessment of utility meters is the process by which compliance of measuring instruments with essential requirements is determined. The uncertainties associated with accuracy measurements during testing shall be evaluated and accounted for when making decisions about conformity with the specifications. This paper describes evaluation methods of measurement results in utility meters testing applicable for conformity assessment and for testing laboratories.
\end{abstract}

Key words: conformity assessment, utility meters, testing, uncertainty

\section{Introduction}

In science and engineering, objects of interest are characterized by measurement and testing. Measurement is defined process of experimentally obtaining quantity values that can reasonably be attributed to a property of a body or substance. Testing is the technical procedure consisting of the determination of characteristics and properties of a given object or process and is performed in accordance with defined test procedures.

The Directive 2014/32/EU of the European Parliament and of the Council of 26 February 2014 on the harmonisation of the laws of the Member States relating to the making available on the market of measuring instruments [1] is aimed at creating a single market for measuring instruments across the European Union. The measurements from the readings of utility meters (water meters, gas meters, active electric energy meters, thermal energy meters, etc.) accurately reflect actual energy consumption and are used to calculate the amount of energy supplied to the customer for the purpose of billing. However, only meter types that operate to defined accuracy and performance requirements can be approved for this purpose.

Whenever the testing is made, it is with the objective of generating data. Data is sequentially analysed and compared with relevant requirements in such a way that an appropriate decision can be taken. In order to utilise a result to decide whether it indicates compliance or non-compliance with a specification, it is necessary to take into account the measurement uncertainty.

\section{Principle measurement results evaluation}

The conformity assessment of utility meters is the process by which compliance of meters with essential requirements is determined. The essential requirements in respect of utility meters, if they are subject to legal metrological control, are defined by Directive 2014/32/EU [1]. The harmonised standards or normative documents (OIML recommendations) are mostly used for conformity assessment and include appropriate tests and test procedures. 
The maximum permissible relative errors (MPE) for the water meters according to Directive 2014/32/EU [1] for water having a temperature $\leq 30^{\circ} \mathrm{C}$ is shown in Figure 1.

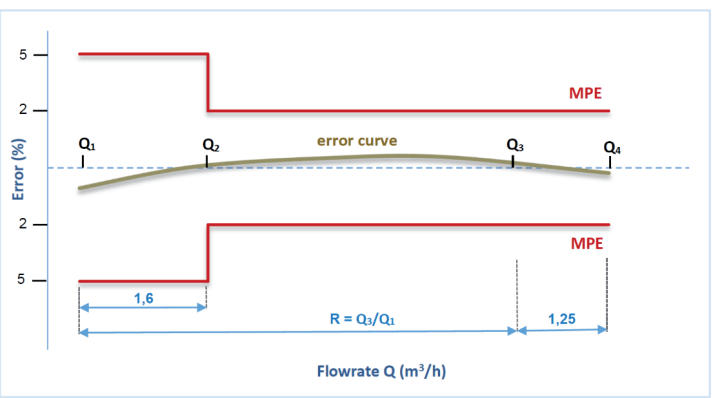

Figure 1: Illustration of the maximum permissible errors for the water meters with water having a temperature $\leq 30{ }^{\circ} \mathrm{C}$ according to Directive 2014/32/EU.

The use of recognized standard procedures EN ISO 4064-2 [2] or OIML R 49-2 [3] for the conformity assessment according to module B (EU-type examination) eliminate many potential sources of measurement uncertainty. Definitions, calculations, and other information necessary to evaluate the test data are contained in such test procedures. Measurement uncertainty enables users of a measured quantity value to make comparisons, in the context of conformity assessment, to obtain the probability of making an incorrect decision based on the measurement, and to manage the consequential risks.

We carried out the tests for determination of volume indication errors by using a testing method in which the quantity of water passed through a water meter was collected in collecting vessels and the quantity was determined volumetrically. The error curve in depending on flowrates obtained from the experimental measuring tests of volumetric rotary piston cold water meter (nominal diameter DN 15, temperature class T50, Q3 $=2.5 \mathrm{~m}^{3} / \mathrm{h}, \mathrm{R}=160$ ) at the reference water temperature $20^{\circ} \mathrm{C}$ according to EN ISO 4064-2 [2] is shown in Figure 2. For better illustration there is drawn the error curve within flow rate zone $(\mathrm{Q} 2 \leq \mathrm{Q} \leq \mathrm{Q} 4)$. The maximum permissible relative error (MPE) of the water meters according to Directive 2014/32/EU [1] for the upper flow rate zone (Q2 $\leq \mathrm{Q} \leq \mathrm{Q} 4)$ is $\pm 2 \%$ with water having a temperature $\leq 30^{\circ} \mathrm{C}$

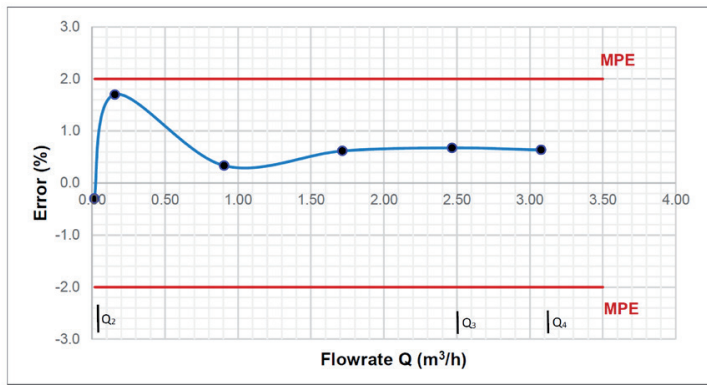

Figure 2: The error curve of the water volume indication in depending on flowrates obtained from the tests of volumetric rotary piston cold water meter

\section{Results evaluation approach by expanded uncertainty of measurement}

Usually the uncertainty of the measurement is evaluated by the calculation of the standard uncertainty of the output estimate and expanded uncertainty of measurement. For a random variable the variance of its distribution or the positive square root of the variance, called standard deviation, is used as a measure of the dispersion of values. The standard uncertainty of measurement associated with the output estimate or measurement result $y$, denoted by $u(y)$, is the standard deviation of the measurand $Y$. It is to be determined from the estimates $x_{i}$ of the input quantities $X_{i}$ and their associated standard uncertainties $u\left(x_{i}\right)$ [4].

For uncorrelated input quantities the square of the standard uncertainty associated with the output estimate $\mathrm{y}$ is given by equation:

$u^{2}(y)=\sum_{i=1}^{N} u_{i}^{2}(y)$

The quantity $u_{i}(y)$ is the contribution to the standard uncertainty associated with the output estimate $y$ resulting from the standard uncertainty associated with the input estimate $x_{i}$ according to equation:

$u_{i}(y)=c_{i} u\left(x_{i}\right)$

where $c_{i}$ is the sensitivity coefficient associated with the input estimate $x_{i}$ calculated by the equation:

$c_{i}=\frac{\partial f}{\partial x_{i}}=\frac{\partial f}{\partial X_{i}}\left(X_{1}=x_{1} \ldots X_{N}=x_{N}\right)$

The uncertainty of measurement associated with the input estimates is evaluated according to Type A and Type B method of evaluation. The Type A 
evaluation of standard uncertainty is the method of evaluating the uncertainty by the statistical analysis of a series of observations. The standard uncertainty $u(q)$ associated with the input estimate $q$ is the experimental standard deviation of the mean expressed as follows:

$$
u(\overline{\mathrm{q}})=s(\overline{\mathrm{q}})
$$

where the experimental variance of the mean is given by the equation:

$s^{2}(\overline{\mathrm{q}})=\frac{s^{2}(q)}{\mathrm{n}}$

The Type B evaluation of standard uncertainty is the method of the uncertainty associated with an estimate $x_{i}$ of an input quantity $X_{i}$ by means other than the statistical analysis of a series of observations. The standard uncertainty $u\left(x_{i}\right)$ is evaluated by scientific judgement based on all available information on the possible variability of $X_{i}$.

Within European co-operation for accreditation rules the laboratories state an expanded uncertainty of measurement $U$, obtained by multiplying the standard uncertainty $u(y)$ of the output estimate $y$ by a coverage factor $k$, as follows:

$$
U=k u(y)
$$

In case where a normal (Gaussian) distribution can be attributed to the measurand and the standard uncertainty associated with the output estimate has sufficient reliability, the standard coverage factor $k=2$ shall be used. The assigned expanded uncertainty corresponds to a coverage probability of approximately $95 \%$.

In the case small number repeated observations it is recommended to estimate the reliability of the standard uncertainty by its effective degrees of freedom. The effective degrees of freedom $v_{\text {eff }}$ of the standard uncertainty $u(y)$ associated with the output estimate y can be calculated from following the Welch- Satterthwaite formula:

$$
v_{e f f}=\frac{u^{4}(y)}{\sum_{i=1}^{N} \frac{u_{i}^{4}(y)}{v_{i}}}
$$

Coverage factors derived from effective degrees of freedom is than based on a t-distribution evaluated for a coverage probability of $95.45 \%$.

We took into consideration following sources of uncertainty regarding determination of volume indication errors by using a testing method in which the quantity of water passed through a water meter was determined volumetrically:

» water meter scale reading

" measurement repeatability

"reference standard

" water temperature of the reference standard

" coefficient of cubical thermal expansion of the reference standard material

» coefficient of cubical thermal expansion the water

» meniscus reading of reference standard

" evaporation of the water

The calculated values of the volume indication relative errors in depending on flowrates (see Figure 2) including illustration of expanded uncertainty of measurement $U$ is shown in Figure 3.

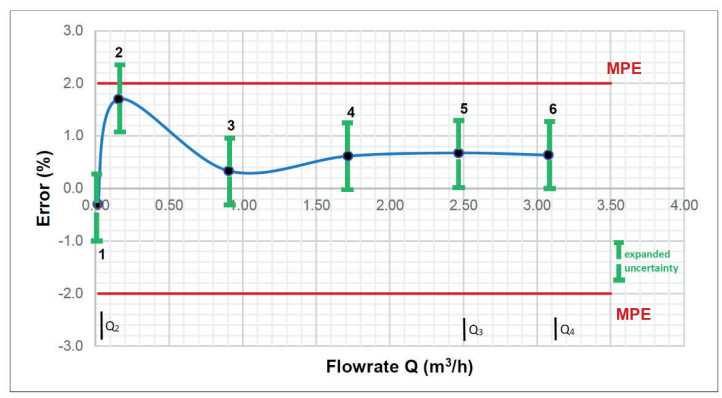

Figure 3: The values of the water volume indication errors in depending on flowrates with expanded uncertainty of measurement $U$

When compliance with a specification is made according to EN ISO/IEC 17025 [5] it should be clear which coverage probability for the expanded uncertainty $U$ has been used. The compliance is accepted in the case that following condition is fulfilled [6]:

$$
E_{m(i)} \pm U \leq M P E
$$

where: $\mathrm{E}_{m(i)}$ - is the relative error of indication at a flow rate $\mathrm{i}(\mathrm{i}=1,2 \ldots \mathrm{n})$; $\mathrm{U}$ - is expanded uncertainty of measurement; MPE - is the maximum permissible relative error at a flow rate $\mathrm{i}(\mathrm{i}=1,2 \ldots \mathrm{n})$.

On the basic of experimentally determined relative errors of the water meter indication and calculated relevant expanded uncertainty of measurement $U$ the notified body should pronounce a statement that the tested water meter is not in line with the requirement of the Directive 2014/32/EU [1] for MPE because as it follows from 
the Figure 3 , within the flowrates from $Q_{2}$ to $Q_{4^{\prime}}$ in the tested flowrate point 2 the sum of the relative error of indication and expanded uncertainty of measurement is above MPE (MPE $=2 \%)$.

\section{Results evaluation approach by Best Measurement Capability}

WELMEC European Cooperation in Legal Metrology [7] defined different approach regarding acceptance criteria for accuracy measurements of utility meters during conformity assessment according to Directive 2014/32/EU [1]. In order to obtain reliable results for conformity assessment purposes, the traceability and the Best Measurement Capability (BMC) of the test equipment including method used during conformity assessments shall be known. The Best Measurement Capability is the expended uncertainty (coverage factor $k=2$ ) of the measurand without the uncertainty contribution of the measuring instrument under test.

For the conformity assessment according to module B (EU-type examination) it is recommended fulfilment following equation [7]:

$$
B M C<\frac{1}{5} M P E
$$

In the case fulfilment of the condition stated in equation (9), the compliance is accepted when following condition is fulfilled:

$$
E_{m(i)}<M P E
$$

From the equation (10) follows that the relative error of indication $\mathrm{E}_{m(i)}$ is directly compared with MPE. This principle of measuring results evaluation is called also as "share risk" regarding acceptable level of the risk due to the uncertainty. By means of this principle the uncertainty is not taken into account when deciding on the conformity.

In the case that the condition stated in equation (9) is not fulfilled, the testing results should be evaluated by using the equation (8).

The calculated values of the volume indication relative errors in depending on flowrates (see Figure 2) including illustration of BMC as the expended uncertainty of the measurand without the uncertainty contribution of the measuring instrument under test is shown in Figure 4.

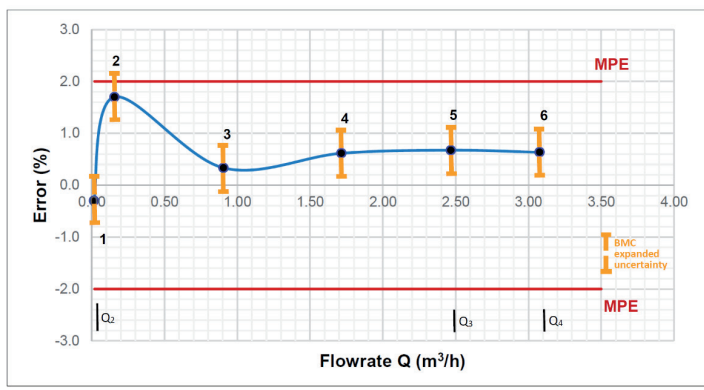

Figure 4. The values of the water volume indication relative errors in depending on flowrates with BMC expanded uncertainty

On the basic of experimentally determined relative errors of the water meter indication and calculated relevant BMC expanded uncertainty the notify body following the share risk principle should pronounce a statement, that the tested water meter is in line with the requirement of the Directive 2014/32/EU for MPE within the flowrates from $Q_{2}$ to $Q_{4}$ even in tested flowrate point 2 (see Figure 4) at which, the sum of the relative error of indication and BMC expanded uncertainty is above MPE (MPE $=2 \%$ )

\section{Conclusions}

The uncertainties associated with accuracy measurements of utility meters during testing shall be evaluated and accounted for when making decisions about conformity in the context of conformity assessment. This is mainly because test uncertainty can increase the risk of making incorrect decisions, such as failing a conforming instrument or passing a non-conforming instrument when the test result is close to a specification limit (MPE). This is important for Notified Bodies to have a uniform way of handling uncertainty so the risk for the manufacturer of a measuring instrument is the same regardless of which Notified Body is used.

We recommend to evaluate testing results for the purposes of conformity assessment of utility meters by using Best Measurement Capability expanded uncertainty. Harmonised standards or normative documents (OIML recommendations) include relatively exact requirements for the test equipment, facilities and methods in relation to testing of utility meters and thereby create applicable conditions for this uniform approach. 


\section{Acknowledgement}

This research was funded by APVV-17-0258 Digital engineering elements application in innovation and optimization of production flows. APVV-19-0418 Intelligent solutions to enhance business innovation capability in the process of transforming them into smart businesses. KEGA 001TUKE-4/2020 Modernizing Industrial Engineering education to Develop Existing Training Program Skills in a Specialized Laboratory. VEGA 1/0438/20 Interaction of digital technologies to support software and hardware communication of the advanced production system platform.

\section{References}

1. Directive 2014/32/EU of the European Parliament and of the Council of 26 February 2014 on the harmonisation of the laws of the Member States relating to the making available on the market of measuring instruments (recast) issued by the European Parliament and the Council of the European union, $102 \mathrm{p}$.

2. EN ISO 4064-2: 2014, Water meters for cold potable water and hot water. Part 2: Test methods, issued by European Committee for Standardization.

3. OIML R 49-2: Edition 2013 (E), Water meters for cold potable water and hot water. Part 2: Test methods, issued by International organization of legal metrology, issued in 2013, $112 \mathrm{p}$.

4. EA-4/02 M: 2013, Evaluation of the Uncertainty of Measurement In Calibration, issued by European cooperation for Accreditation, issued in 2013, 75 p

5. EN ISO/IEC 17025:2017, General requirements for the competence of testing and calibration laboratories.

6. ILAC-G8:09/2019, Guidelines on Decision Rules and Statements of Conformity, issued by International Laboratory Accreditation Cooperation, in 2019, 20 p.

7. WELMEC 11.1: 2017 Measuring Instruments Directive 2014/32/EU. Common application for utility meters, issued by WELMEC European Cooperation in Legal Metrology, 26 p. 